\title{
Mutation rates in hybrids between sibling species of Drosophila
}

\author{
Jerry A. Coyne
}

Department of Ecology and Evolution, The University of Chicago, 1103E. 57th Street, Chicago, Illinois 60637 U.S.A.

It has recently been suggested that sterility or inviability in species hybrids might result from the movement of transposable elements. Because such movement is often detectable by an increased mutation rate, I studied the effect of interspecific hybridization in the Drosophila melanogaster group on the mutation rate of $\mathrm{X}$-linked visibles. This rate did not differ between hybrids and intraspecific controls. This was also true for the germ-line excision rate of a transposable element, although the rate of somatic excision was two to sixfold higher in hybrids than in pure species. Combined with previous work, these results do not support a role for transposable elements in speciation.

\begin{abstract}
"At present we are completely ignorant of the evolutionary importance of transposable element systems, relative to other genetic mechanisms, for generating diversity and promoting speciation."
\end{abstract}

(Kidwell, 1982, p. 148)

\section{INTRODUCTION}

The discovery of a new genetic phenomenon is often followed by the proposal that it may solve a long-standing evolutionary problem. One example is the recent suggestion that transposable elements may cause speciation.

Present in many species (Shapiro, 1983), these elements are usually stationary but in some cases show bursts of excision and transposition. In Drosophila melanogaster, massive transposition occurs when males containing complete elements of the P or I families are crossed to females from strains lacking them. Offspring of such a cross show a syndrome of abnormalities collectively called hybrid dysgenesis, which includes temperature-sensitive gonadal atrophy or egg lethality, male recombination (usually absent in Drosophila), and elevated rates of chromosome breakage and germ-line mutation (Picard, 1976; Kidwell et al., 1977). These phenomena are almost certainly related to the movement of transposons, although it is not yet clear how such movement causes sterility and gonadal atrophy.
Some interspecific hybrids show anomalies similar to those seen in hybrid dysgenesis. Gonadal atrophy and/or sterility is a common form of postzygotic isolation among species, frequently the only anomaly observed in hybrids. Some Drosophila hybrids show elevated rates of mutation and chromosome breakage (Sturtevant, 1939; Naveira and Fontdevila, 1985 and references therein), and novel electrophoretic alleles have been reported in hybrid zones (Gould and Woodruff, 1978; Sage and Selander, 1979).

The parallels between dysgenic hybrids in $D$. melanogaster and species hybrids have led to the suggestion that speciation might be caused by high rates of transposition. First proposed by Engels and Preston (1979) and Kidwell and Novy (1979), this idea was reviewed by Rose and Doolittle (1979) and also discussed by Martin et al. (1983), Bregliano and Kidwell (1983), Kidwell (1983), Bingham et al. (1982), and Ginzburg et al. (1984). Bregliano and Kidwell (1983, p. 403) explain the hypothesis: "A second way that hybrid dysgenesis determinants may have implications for evolution is by acting as postmating reproductive isolating mechanisms. Crosses between long-established laboratory strains and natural population strains tend to produce hybrids with lower relative fitness than the parental strains. Such hybrids may be similar to those produced in nature when population boundaries meet again after a period of geo. 
graphical isolation. Studies with hybrid dysgenesis suggest that populations may evolve rapidly as carriers of transposable elements, and these may act, separately or in conjunction with other isolating mechanisms, to promote reproductive isolation and eventual speciation.... It is interesting to note that there are many examples of intersubspecific and interspecific crosses in the genus Drosophila that have parallels with hybrid dysgenic crosses (see Kidwell, 1983 for a review)". Ginzburg et al. (1984), who mathematically model transposoninduced speciation, speculate that (p. 339) "Transposable elements may be the central biological agents controlling much of the evolutionary process in sexually reproducing organisms, including the generation of mutational diversity and reproductively isolated lines of descent involved in speciation ... our view of the process of speciation predicts that closely related species should carry transposon families that distinguish them and that such families should be causally related to the partial or full sterility in hybrids between the two species".

The first prediction is correct: closely related Drosophila species sometimes have different families of elements (Martin et al., 1983; Brookfield et al., 1984). It is much more difficult, however, to demonstrate that their movement causes hybrid inviability or sterility, as this is not known for certain even in hybrid dysgenesis within D. melanogaster. If transposable elements do, however, move pervasively in hybrids, this should be detectable by increases in the rates of mutation and chromosome breakage. Naveira and Fontdevila (1985) did observe high rates of chromosome breakage in $D$. buzzatii/D. serido hybrids; but studies of mutation rates have given mixed results, with reports of both elevated rates (Sturtevant, 1939; Miller, 1950) and normal rates (Otis, 1947; Hey, 1988) in species hybrids.

Here I investigate mutation rates in hybrids among four closely related species of Drosophila. All hybridizations among these species produce some sterile or inviable offspring, offering the possibility of transposon-induced reproductive isolation. I measured not only the rate of X-linked visible mutations arising in the germ line of hybrid females, but also the rate of movement in hybrids of a transposon known to excise at high frequencies within one species. Increased mutation rates in hybrids may reflect not only the movement of such transposons, but also any genetic divergence between species in the mechanisms that repair genetic material. Sturtevant (1939) suggested that different species would evolve different genetic pathways for such repair, whose breakdown in hybrids might increase the mutation rate.

\section{METHODS AND MATERIALS}

This study used four sibling species in the $D$. melanogaster subgroup: $D$. melanogaster, $D$. sechellia, D. simulans, and D. mauritiana (most of the work concentrated on simulans/mauritiana hybrids). D. melanogaster and $D$. simulans are worldwide human commensals, while the other two species are endemic to different archipelagos in the Indian Ocean. The females of all four species are morphologically identical, while males can be distinguished only by the posterior process of the genital arch (Tsacas and David, 1979; Tsacas and Bächli, 1981). Electrophoretic, DNA, and karyotypic evidence show that $D$. simulans, $D$. mauritiana, and $D$. sechellia form a closely related group to which $D$. melanogaster is an outgroup, but the phylogeny of the three ingroup species is unresolved (Coyne and Kreitman, 1986; Caccone et al., 1988; Lachaise et al., 1988). The three ingroup species can be crossed reciprocally to yield fertile females but sterile males. The fertile $F_{1}$ females can be readily backcrossed to either parental species, yielding fertile females and semisterile males. When crossed to $D$. melanogaster, however, these three species produce unisexual progeny that are always sterile.

\section{$X$-linked mutation rate}

In a study similar to that of Sturtevant (1939), I compared anomalies in the progeny of hybrid females with those in progeny from a pure species. Hybrid females were produced by crossing $D$. simulans females from an isofemale strain (Florida City, Florida; see below) to males from an isofemale line of $D$. mauritiana (National Drosophila Species Resource Center, Bowling Green State University, Bowling Green, Ohio). These hybrid females were then backcrossed to male $D$. simulans containing the $\mathrm{X}$-linked mutations yellow $(y, 1-0)$, white $(w, 1-4 \cdot 1)$, and forked $(f, 1-56 \cdot 0)$. Any X-linked mutations arising in hybrid females will appear in their male offspring, while mutations at $y, w$, or $f$ will also appear in females (Green (1977) reported that hybrid dysgenesis in $D$. melanogaster raises mutation rates at the yellow locus). As a control, females from the Florida City strain of $D$. simulans were crossed to $D$. simulans $y, w, f$ males.

Flies were lightly etherized and examined for abnormalities in eye colours and textures, body 
colours, wing veination, and major bristle patterns (scutellar and dorsocentral). Eye and wing anomalies were scored as putative mutations if they occurred on both sides of the body, while single missing bristles (very rarely found) were not counted as mutations. These traits encompass most of the X-linked morphological mutations described by Lindsley and Grell (1967). Females were examined only for mutations at yellow, white, or forked.

Putative mutants were genetically analyzed to localize them to chromosome and homologize them with existing mutants. Unfortunately, males from backcrosses between simulans and mauritiana are almost always sterile (Coyne 1984), and none of the putative male mutants could be tested. Several were, however, phenotypically similar to well-known mutations in $D$. simulans or D. melanogaster. Female offspring are fertile, so that putative yellow, white, or forked mutations could be preserved and genetically analyzed. These crosses were made in bottles containing Instant Drosophila Food (Carolina Biological Supply Company) and reared at $24^{\circ} \mathrm{C}$.

Mutations appearing in interspecific and control crosses need not have originated in the gametes of female parents, but may have been segregating in the original isofemale strains. This is not a serious problem when one looks for mutation rates as high as those produced by hybrid dysgenesis in D. melanogaster. Hey (1988) estimates dysgenic mutation rates to all $\mathrm{X}$-linked visibles between $0.43 \times 10^{-2}$ and $2 \times 10^{-2}$, while Green (1977) reports rates between $0.5 \times 10^{-3}$ and $1 \times 10^{-3}$ at four $\mathrm{X}$-linked loci. As shown below, the rates in both hybrids and controls (around $10^{-4}$ for all X-linked visibles) were much lower than this and nearly equal to each other. These rather low observed rates also imply that our detected variants were indeed new mutations rather than variants originally present in the isofemale lines, although this conclusion is weak because we do not know the magnitude of selection on such variants or the amount of inbreeding in the lines during laboratory culture. A more rigorous test would involve accumulating mutations on extracted chromosomes, but this is presently impossible because the genetic tools for extraction do not exist in $D$. simulans, D. mauritiana, or D. sechellia.

\section{White-peach mutation rate}

The transposable element mariner was discovered by its insertion into the white locus of $D$. mauritiana, producing a yellowish eye-color muta- tion known as white-peach $\left(w^{p c h}\right)$. Hartl and his colleagues have isolated, sequenced, and characterized this transposon (see summary in Hartl, 1989). Mariner occurs in 20-30 copies in $D$. mauritiana, 2 copies in D. simulans, and 1 or 2 copies in D. sechellia, and is also found in other species of the $D$. melanogaster group. It has not, however, been found in D. melanogaster (Hartl, 1989).

Unlike most transposons, mariner has a high rate of both germ-line and somatic excision within strains. Germ-line movement, detected by the production of offspring with wild-type or white eyes by white-peach parents, occurs at a rate of $1 \times$ $10^{-4}-4 \times 10^{-4}$ per gamete per generation, with the rate about twice as high in males as in females. Somatic excision, detected as spots of wild-type pigment in a white-peach eye, occurs at a rate of about $4 \times 10^{-3}$ in both sexes. Movement of mariner elements has been directly implicated in germ-line and somatic mutation (Hartl, 1989).

Haymer and Marsh (1986) reported that germline excision of mariner is significantly higher in D. simulans/D. mauritiana hybrids than in pure D. mauritiana. This difference, however, was observed only in male progeny, and there was no difference between hybrids and controls in the rate of somatic excision. These preliminary results suggest that the element may move more often in species crosses. To investigate this sytematically, I made a variety of crosses among the following strains:

\section{D. mauritiana}

white-peach $\left(w^{\text {pch }}\right)$ Strain (obtained from Dr Daniel Hartl) carrying the mariner element inserted in the white locus.

white $(w) \quad$ Homologous to D. melanogaster white, this mutation originated in an originated in isofemale line maintained by R. C. Woodruff.

synthetic (syn) A strain produced by combining six isofemale lines collected by $\mathrm{O}$. Kitagawa on Mauritius in 1981. A single isofemale line from the same collection as above, but not used to make the synthetic strain.

A single isofemale line from the same collection as above, but not used to make the synthetic strain. 
D. simulans

white-Riverside $\left(w^{R}\right)$ Allele of white, producing pure white eyes. Mutation arose in a wild stock collected in Riverside, California by A. Hoffman and $M$. Turelli (Inoue and Yamamoto, 1987).

white-Saito $\left(w^{S}\right)$

white-apricot $\left(w^{a p}\right)$

Allele of white, producing pure white eyes. Derived from a stock collected in Tokyo, Japan by K. Saito (Inoue and Yamamoto, 1987).

Allele of white, identical in appearance to $w^{a p}$ of $D$. melanogaster. Obtained from M. M. Green.

FC

Isofemale line of $D$. simulans collected in Florida City, Florida in 1985.

D. melanogaster white $(w)$

\section{D. sechellia}

sec

Obtained from MidAmerica Drosophila stock Center, Bowling Green State University, Bowling Green, Ohio.

An isofemale line obtained from Dr Hugh Robertson.

All of these crosses were reared on cornmealmolasses-agar food at $24^{\circ} \mathrm{C}$. The eyes of all progeny were inspected under a $20 \times$ dissecting microscope, which easily revealed a single red ommatidium in an otherwise white-peach eye. Germ-line mutations were revealed as complete color changes of both eyes from white-peach to white or red (no other colours were seen) while somatic mutations were scored as patches of red colouration-as small as a single ommatidium-in an otherwise white-peach eye. All putative germ-line mutations were genetically analyzed, although some interspecific male hybrids were sterile and could not be tested. Heritability experiments revealed whether genetic differences among individuals affected the probability of somatic variegation.

\section{RESULTS}

\section{$X$-linked mutations}

Of 27,063 males scored from the backcross of hybrid mauritiana/simulans females to simulans $y, w, f$ males, ten had bilateral anomalies resembling mutations: four with an eye phenotype resembling lozenge-sparkling, two with roughened eyes, two with a yellowish-body colour darker than yellow but resembling yellow-2, one with short, thin bristles, and one with a distal fusion of two longitudinal wing veins. All of these males were sterile when crossed to $D$. simulans attached-X females. The maximum possible frequency of $\mathrm{X}$-linked mutations in these hybrid females is thus $10 / 27,063$ or $3.7 \times 10^{-4}$.

The control cross of simulans Florida City females to simulans $y, w, f$ males also produced ten anomalous males. Two had the lozengesparking phenotype (one proved to be $l z^{s p}$ and the other a non-genetic phenocopy), one had purple eyes that proved to be the X-linked mutation ruby, two had truncated wings (both of which were new X-linked mutations), one had "singed"-like dorsocentral bristles (a nongenetic phenocopy), one had net wings (an autosomal mutation), one had the X-linked yellow-2 mutation, one had a new $\mathrm{X}$-linked mutation producing scalloped wings, and had the X-linked mutation garnet. Although three of these individuals did not carry X-linked mutations, the appropriate comparison with the interspecific cross (in which genetic tests were not possible) involves the total frequency of anomalies, which in the intraspecific cross is $10 / 22,886$ or $4.4 \times 10^{-4}$. The frequencies of possible germ line anomalies do not differ between control and interspecific crosses $\left(\mathrm{G}_{1}=0 \cdot 14, P>0 \cdot 7\right.$; Sokal and Rohlf 1981).

Two mutants were found among 31,038 interspecific backcross females scored for the three X-linked tester loci. One had the yellow-2 body-colour phenotype, and the other white eyes. Both proved to be genetic mutations that were stable in pure culture (no revertants seen among 10,985 white flies and 10,253 yellow flies). Among 24,175 females in the intraspecific control, we found one female with the yellow-2 phenotype, who died before producing offspring. Combining the mutations in females with those in males, the observed mutation rate at yellow is $3 / 58,101$ or $5.2 \times 10^{-5}$ for the interspecific cross and $2 / 47,061$ or $4.2 \times 10^{-5}$ in the intraspecific control. These rates are not statistically different $\left(G_{1}=0.05, P>0.75\right)$. There is obviously no difference between experimental and control in the mutation rate at the white locus, for only one mutation was seen.

There is no obvious increase in the mutation rate to X-linked visibles in the germ line of species hybrids when compared to pure species. 
Table 1 Offspring of crosses scored for somatic and germ-line mutations. Somatic and germ-line mutation rates are calculated per $w^{p c h}$ chromosome, not per individual; we have assumed that these rates are independent of whether individuals contain one or two such chromosomes. Crosses are given with female parents first; e. g. $\left(w^{p c h} \times \operatorname{sim}\right) \times w^{p c h}$ indicates a backcross between hybrid females (offspring of mauritiana $w^{p c h}$ females and simulans males) and mauritiana $w^{p c h}$ males

\begin{tabular}{|c|c|c|c|c|c|c|}
\hline & \multicolumn{3}{|c|}{ Male offspring } & \multicolumn{3}{|c|}{ Female offspring } \\
\hline & Total & Somatic (rate) & Germ (rate) & Total & Somatic (Rate) & Germ (rate) \\
\hline 1. $w \times w^{p c h}$ & - & - & - & 5094 & $59(0 \cdot 012)$ & $7(0.0014)$ \\
\hline 2. $w^{p c h} \times w$ & 2068 & $134(0.065)$ & $2(0.0010)$ & 2577 & $70(0.027)$ & $3(0 \cdot 0012)$ \\
\hline 3. $w^{p c h} \times \operatorname{syn}$ & 6746 & $17(0.002)$ & $5(0.0007)$ & - & - & - \\
\hline 6. $\left(175 \times w^{p c h}\right) \times w^{p c h}$ & 614 & $29(0.047)$ & $0(0 \cdot 0000)$ & 705 & $23(0.016)$ & $0(0.000)$ \\
\hline \multicolumn{7}{|l|}{ Interspecific crosses } \\
\hline 7. $w(\mathrm{mel}) \times w^{p c h}$ & - & - & - & 2073 & $184(0.089)$ & $1(0 \cdot 0005)$ \\
\hline 8. $w^{p c h} \times \sec$ & 130 & $33(0 \cdot 254)$ & $0(0.0000)$ & - & - & - \\
\hline 9. $\left(\sec \times w^{p c h}\right) \times w^{p c h}$ & 967 & $128(0 \cdot 132)$ & $0(0.0000)$ & 1071 & $119(0.056)$ & $0(0 \cdot 0000)$ \\
\hline 14. $\left(\operatorname{sim} \times w^{p c h}\right) \times w^{p c h}$ & 3168 & $144(0.046)$ & $2(0.0006)$ & 3482 & $115(0.016)$ & $0(0.0000)$ \\
\hline 15. $\left(w^{a p} \times w^{p c h}\right) \times w^{a p}$ & 2373 & $193(0.081)$ & $3(0.0013)$ & 3168 & $113(0.036)$ & $5(0.0016)$ \\
\hline
\end{tabular}

\section{White-peach mutations}

Table 1 gives the frequencies of somatic and germline mutations at white-peach in hybrids and controls. Although some backcross males with a putative germ-line mutation were sterile and could not be tested, all putative mutants that were fertile in both control and experimental crosses were indeed germ-line mutations. We therefore assume that the sterile revertants were also germ-line mutations.

The rates of germ-line mutation are low in both sets of crosses. The total rate for all intraspecific crosses $(25,209$ individuals examined $)$ is $7 \cdot 1 \times 10^{-4}$ mutations $/ w^{p c h}$ allele. This is close to the range of $1 \times 10^{-4}$ to $4 \times 10^{-4}$ reported by Hartl (1989), and there is no statistical heterogeneity among crosses. The rate of germ line mutation in interspecific crosses is $6 \cdot 7 \times 10^{-4}$ (15,000 individuals examined), also with no heterogeneity among crosses. Obviously, the rates of inter- and intraspecific reversion do not differ when the sexes are considered separately or together $\left(G_{1}=0.03, P>0.8\right.$ for the latter comparison). Although the frequency of white-peach excision in the germ line is fairly high, it is no higher in interspecific than in intraspecific crosses.

The rates of somatic mutation, on the other hand, are higher in interspecific hybrids than in controls (table 1). There is large variation in the frequency of eye variegation among intraspecific crosses as well as among interspecific crosses, with frequency of variegation per allele ranging from 0.002 to 0.065 in the former set and from 0.033 to 0.243 in the latter. This heterogeneity is statistically significant when the sexes are tested separately in both sets of crosses $\left(P<10^{-4}\right.$ in all four $G$ tests $)$. Nevertheless, the rates of variegation are clearly higher among interspecific crosses: all six of the highest variegation rates were seen in hybrids.

The heterogeneity of values within each set of crosses makes statistical comparison of intraversus interspecific crosses difficult, particularly because the highest interspecific rates occur in those hybridizations yielding the fewest progeny, when the white-peach allele is introduced through the $D$. mauritiana mother (these crosses are very difficult). A conservative nonparametric test on the unweighted rates of somatic variegation seems most appropriate, and shows that these rates are significantly higher in inter- than in intraspecific crosses for both males (Mann--Whitney $U^{\prime}=28$, $Z_{\text {corr }}=2.37$, two-tailed $P=0.02$ ) and females (Mann-Whitney $U^{\prime}=26, Z_{\text {corr }}=-2 \cdot 27,0 \cdot 02<P<$ $0 \cdot 05$ ). (The data from all hybridizations were used because somatic variegation occured in a hybrid genome). This weak test masks the large difference in average rate between intra- and interspecific crosses, the former being 0.013 reversions/allele in males and 0.016 in females (three or four times the values reported by Hartl (1989) for $D$. 
Table 2 Test for heritability of somatic variegation. Variegated and non-variegated white-peach females from the crosses shown were backcrossed to $D$. mauritiana white-peach males, and somatic variegation scored among the offspring. Probabilities are one-tailed

\begin{tabular}{|c|c|c|c|c|c|c|}
\hline $\begin{array}{l}\text { Cross producing } \\
\text { mothers }\end{array}$ & \multicolumn{2}{|c|}{$\frac{\text { Male offspring }}{w^{p c h}}$} & \multicolumn{2}{|c|}{$w^{p c h} / w^{p c h}$} & \multicolumn{2}{|l|}{$w / w^{p c h}$} \\
\hline \multicolumn{7}{|l|}{$\left(\sec \times w^{p c h}\right) \times w^{p c h}$} \\
\hline $\mathrm{G}$ value & & $6 \cdot 12^{* *}$ & & $7 \cdot 15^{* *}$ & & $5 \cdot 12^{*}$ \\
\hline \multicolumn{7}{|l|}{$w^{\mathrm{S}} \times w^{p c h}$} \\
\hline Variegated mothers & 532 & $0 \cdot 130$ & 594 & 0.099 & - & - \\
\hline Nonvariegated mothers & 692 & 0.069 & 722 & 0.074 & - & - \\
\hline $\mathrm{G}$ value & & $13 \cdot 8^{*}$ & & $2 \cdot 50(p=0.06)$ & & \\
\hline
\end{tabular}

$* P<0.025$.

** $P<0.01$.

mauritiana); and the latter 0.078 in males and 0.039 in females. Our results are the opposite of those reported by Haymer and Marsh (1986), who found a higher rate of germ-line but not somatic transposition in species crosses.

Several other points are worth noting. First, the rate of somatic variegation offspring is higher in male than in female in all seven crosses in which both sexes could be scored (sign test: $P=0 \cdot 016$ ), although Haymer and Marsh (1986) report no difference between the sexes. Second, in both pairs of reciprocal crosses permitting measurement of somatic variegation (crosses 1 vs. 2 and 13 vs. 14 in table 1), the rates are significantly higher when the white-peach allele is introduced through the mother $\left(P<10^{-4}\right.$ for each of the three possible comparisons among the sexes). Because Hartl (1989) reports that somatic variegation rates do not differ between reciprocal crosses, this phenomenon deserves further investigation, particularly because the highest rate occurs when the transposable element is introduced through females. This is the opposite of what is observed for I-R and P-M hybrid dysgenesis in $D$. melanogaster (Bregliano and Kidwell, 1983).

Two sets of interspecific crosses investigated the heritability of somatic variegation. Variegated and nonvariegated $w^{p c h}$ females from crosses involving $D$. mauritiana and $D$. simulans or $D$. sechellia (crosses 9 and 11 in table 1) were backcrossed to $w^{p c h}$ males, and the frequency of variegation measured among white-peach progeny. In four of the five comparisons between individuals of the same sex from variegated versus nonvariegated mothers (table 2), the offspring of variegated mothers had significantly higher frequencies of variegation. (In the other comparison, the probability bordered on significance under a one-tailed test.) Some heritable factors apparently increase the probability of variegation. Because the species origin of the cytoplasm was identical within each comparison, the difference is probably caused by segregating nuclear genes. Bryan et al. (1987) report a dominantly-acting autosomal factor in $D$. mauritiana causing somatic variegation in all of its carriers; and Medhora et al. (1988) show that this factor, called Mos, is almost certainly a copy of mariner. Our results do not show this all-or-none inheritance, but only frequency differences of a few per cent. Hartl (1989), however, reports that normal somatic variegation within $D$. mauritiana is not heritable.

Finally, a backcross between simulans/ mauritiana hybrid females and $D$. simulans males (data not presented) showed no association of somatic variegation with female sterility, so excision in the soma has no apparent effect on the germ line.

\section{DISCUSSION}

I find no evidence for elevated mutation rates in the germ line of interspecific hybrids. The observed rate for X-linked visibles in hybrids, $3.7 \times 10^{-4}$, probably overestimates the true rate because some of the morphological anomalies in hybrid males may have been either phenocopies or segregants in the original strains. The 95 per cent upper confidence limit for this rate is $5.7 \times 10^{-4}$, almost 
an order of magnitude lower than the lowest mutation rate of $\mathrm{X}$-linked visibles seen in hybriddysgenic crosses within D. melanogaster (Hey, 1988).

The rate of germ-line excision of the mariner element is also nearly identical in intra- and interspecific crosses. The 95 per cent confidence limits for this difference show that it is unlikely to be larger than $5 \times 10^{-4}$, so that a doubling or tripling of the rate in hybrids-as seen for the rate of somatic mutations at $w^{p c h}$-could easily have been detected.

The only mutation rate higher in hybrids than in pure species is that of somatic movement of mariner in the white-peach eye. In some interspecific crosses this rate is nearly 25 per cent per allele per generation, six times higher than that observed in any intraspecific cross and one of the highest rates observed for any transposable element. Based on the observations of significant heterogeneity of variegation rate among crosses within species, of high rates in interspecific crosses regardless of the direction of the cross, and of the heritability of somatic variegation, we suspect that this variegation is affected by nuclear genes differing among species. In addition, the significant differences among reciprocal crosses also imply an influence of the cytoplasm. The cause of this, like that of enhanced somatic variegation in hybrids, is a mystery. Nevertheless, somatic mutationsand changes in their rate-are probably unimportant in the evolution of animals.

Elevated rates of somatic variegation were seen in hybridizations among all pairs of species, and may be a general property of the mariner transposon. The fact that the highest rates occur in crosses with $D$. melanogaster, which contains no copies of mariner, suggests that the rate of somatic excision and transposition may be regulated by the number of copies in the genome. The other notable result is an apparent reciprocal-cross effect on somatic variegation, so that higher rates are seen when both transposon and cytoplasm are derived from the white-peach strain. This has not been described for any other transposon.

With the exception of somatic variegation of white-peach, then, all attempts to show the mobilization of transposons in species crosses have been fruitless, and such movement is unlikely to cause hybrid sterility and inviability. The parallels between gonadal dysgenesis and P-M hybrid dysgenesis, as well as between hybrid sterility and I- $R$ sterility, have led to searches for symptoms of hybrid dysgenesis in species crosses. Early reports of high frequencies of "mutations" in such crosses
(Sturtevant, 1939; Miller, 1950) have, however, been unrepeatable (Otis, 1947; Hey, 1989). Studies of other dysgenic characters such as male recombination and temperature-sensitive gonadal atrophy have also given negative results (Coyne 1985, 1986). Reports of novel alleles in hybrid zones (Gould and Woodruff, 1978; Sage and Selander, 1979) do not necessarily indicate high rates of transposition, for the alleles may result from intragenic recombination. Indeed, the insertion of transposons seems more likely to affect the quantity than the electrophoretic mobility of an enzyme. The strongest evidence for enhanced transposition in any species cross is the high rate of chromosome breakage in $D$. buzzattii/D. serido hybrids (Naviera and Fontdevila, 1985), a study that should be extended to other species.

There is, on the other hand, evidence that transposons are not responsible for postzygotic isolation in nature. The known forms of hybrid dysgenesis in $D$. melanogaster cause sterility in both sexes or in females alone, but in Drosophila the first step in the evolution of postzygotic isolation is its appearance in males (Coyne and Orr, $1989 a, b)$. Sterility in Drosophila hybrids maps repeatedly to specific locations on the $\mathrm{X}$ chromosome (Coyne and Charlesworth, 1986, 1988). This is expected if sterility results from evolution of localized genes, but not if from the movement of transposons. Finally, the sterility and gonadal dysgenesis caused by hybrid dysgenesis is dependent on age or rearing temperature, phenomena which have not been reported in species hybrids (Coyne, 1985).

Although transposons do differ in both number and kind among species, the idea that they cause speciation will not be credible until they can be shown to move at high rates in species hybrids, and that this movement is directly responsible for hybrid sterility. The evidence is against this possibility.

Acknowledgements This work was supported by GM 38462 from the National Institutes of Health. I thank Brian Charlesworth and Dan Hartl for their comments on the manuscript, and Jean David, Mel Green, Dan Hartl, Hugh Robertson, and Masa-Toshi Yamomoto for Drosophila stocks.

\section{REFERENCES}

BINGHAM, P., KIDWELl, M. G. AND RUBIN, G. M. 1982. The molecular basis of P-M hybrid dysgenesis: the role of the P element, a P-strain specific transposon family. Cell, 29, 995-1004. 
BREgliano, J. C. AND KIDWELl, M. G. 1983. Hybrid dysgenesis determinants. In Shapiro, J. A. (ed.) Mobile Genetic Elements, Academic Press, London, pp. 363-410.

BROOKFIELD, J. F. Y., MONTGOMERY, E. AND LANGLEY, C. H. 1984. Apparent absence of transposable elements related to the $\mathrm{P}$ elements of Drosophila. Nature, 310, 330332.

BRYAN, G. J., JACOBSON, J. W. AND HARTL, D. L. 1987. Heritable somatic excision of a Drosophila transposon. Science, $235,1636-1638$.

CACCONE, A., AMATO, D. AND POWEll, J. R. 1988. Rates and patterns of scDNA and mtDNA divergence within the Drosophila melanogaster subgroup. Genetics, 118, 671-683.

COYNE, J. A. 1984. Genetic basis of male sterility in hybrids between two closely related species of Drosophila. Proc. Natl Acad. Sci. USA, 81, 4444-4447.

COYNE, J. A. 1985. Genetic studies of three sibling species of Drosophila with relationship to theories of speciation. Genet. Res., 46, 169-192.

COYNE, J. A. 1986. Meiotic segregation and male recombination in interspecific hybrids of Drosophila. Genetics, 114, 485494.

COYNE, J. A. AND CHARLESWORTH, B. 1986. Location of an $\mathrm{X}$-linked factor causing male sterility in hybrids of Drosophila simulans and D. mauritiana.. Heredity, 57, 243246.

COYNE, I. A. AND CHARLESWORTH, B. 1988. Genetic analysis of $\mathrm{X}$-linked sterility in hybrids between three sibling species of Drosophila. Heredity, 62, 97-106.

COYNE, J. A. AND KREITMAN, M. 1986. Evolutionary genetics of two sibling species, Drosophila simulans and D. sechellia. Evolution, 40, 673-691.

COYNE, J. A. AND ORR, H. A. 1989a. Patterns of speciation in Drosophila. Evolution, 43, 362-479.

COYNE, J. A. AND ORR, H. A. 1989b. Two rules of speciation. In Endler, J. A. and Otte, D. (eds) Speciation and Its Consequences Sinauer and Associates, Sunderland, Ma. (In press).

ENGELS, W. R. AND PRESTON, C. R. 1979. Hybrid dysgenesis in Drosophila melanogaster: the biology of male and female sterility. Genetics, 92, 161-174.

GINZBURG, L. R., BINGHAM, P. M. AND YOO, S. 1984. On the theory of speciation induced by transposable elements. Genetics, 107, 331-341.

GOULD, S. J. AND wOODRUFF, D. S. 1978. Natural history of Cerion. VIII: Little Bahama Bank-a revision based on genetics, morphometrics, and geographic distribution. Bull. Mus. Comp. Zool., 148, 371-415.

GREEN, M. M. 1977. Genetic instability in Drosophila melanogaster: de novo induction of putative insertion mutations. Proc. Natl Acad. Sci. USA, 74, 3490-3493.

HARTL, D. L. 1989. Transposable element mariner in Drosophila. In Berg, D. E. and Howe, M. M. (eds) Mobile DNA, American Soc. Microbiology. (In press).

HAYMER, D. S. AND MARSH, J. L. 1986. Germ line and somatic instability of a white mutation in Drosophila mauritiana due to a transposable genetic element. Develop. Gen., 6, 281-291.

HEY, J. 1988. Speciation via hybrid dysgenesis: negative evidence from the Drosophila affinis subgroup. Genetica. (In press).

INOUE, Y. H. AND M.-T. YAMAMOTO. 1987. Insertional DNA and spontaenous mutation at the white locus in Drosophila simulans. Mol. Gen. Genet., 209, 94-100.
KIDWELL, M. G. 1983. Intraspecific hybrid sterility. In Ashburner, M, Carson, H. L. and Thompson, J. N. Jr. (eds) The Genetics and Biology of Drosophila, vol. 3c, Academic Press, London, pp. 125-154.

KIDWELL, M. G., KIDWELL, J. F. AND SVED, J. A. 1977. Hybrid dysgenesis in Drosophila melanogaster: a syndrome of aberrant traits including mutation, sterility, and male recombination. Genetics, 86, 813-833.

KIDWELL, M. G. AND NOVY, J. B. 1979. Hybrid dysgenesis in Drosophila melanogaster: sterility resulting from gonadal dysgenesis in the P-M system. Genetics, 92, 1127-1140.

LACHAISE, D., CARIOU, M.-L., DAVID, J. R., LEMEUNIER, F., TSACAS, L. AND ASHBURNER, M. 1988. Historical biogeography of the Drosophila melanogaster species group. Evol. Biol., 22, 159-225.

LINDSLEY, D. L. AND GRELL, E. H. 1967. Genetic variations of Drosophila melanogaster. Carnegie Inst. Wash. Pub. 627.

MARTIN, G., WIERNASZ, D. AND SHEDL, P. 1983. Evolution of Drosophila repetitive and dispersed DNA. J. Mol. Evol., 19, 203-213

MEDHORA, M. M., MACPEEK, A. H. AND HARTL, D. L. 1988. Excision of the Drosophila transposable element mariner: identification and characterization of the Mos factor. The EMBO Journal, 7, 2185-2189.

MILLER, D. D. 1950. Observations on two cases of interspecific hybridization with Drosophila athabasca. Amer. Natur, 84, 81-93.

NAVEIRA, H. AND FONTDEVILA, A. 1985. The evolutionary history of Drosophila buzzattii. IX. High frequencies of new chromosome rearrangments induced by introgressive hybridization. Chromosoma, 91, 87-94.

OTIS, E. M. 1947. Mutation rates in Drosophila virilis virilis, Drosophila virilis americana, and their hybrid. Unpublished Ph.D. dissertation, Department of Biology, University of Rochester, New York.

PICARD, G. 1976. Non-Mendelian female sterility in Drosophila melanogaster: hereditary transmission of I factor. Genetics, $83,107-123$.

ROSE, M. R. AND DOOLITTLE, W. F. 1983. Molecular biological mechanisms of speciation. Science, 220, 157-162.

SAGE, R. D. AND SELANDER, R. K. 1979. Hybridization between species of the Rana pipiens complex in central Texas. Evolution, 33, 1069-1088.

Shapiro, J. A. (ed.) 1983. Mobile Genetic Elements. Academic Press, London.

SOKAL, R. R. AND ROHLF F. J. 1981. Biometry. W. H. Freeman and Co., San Francisco.

STURTEVANT, A. H. 1939. High mutation frequency induced by hybridization. Proc. Natl Acad. Sci. USA, 25, 308-310.

TSACAS, L. AND BÄCHLI, G. 1981. Drosophila sechellia n. sp., huitième espèce du sousgroupe melanngaster des Iles Sèchelles (Diptera, Drosophilidae). Rev. Fr. Entomol., 3, $146-150$

TSACAS, L. AND DAVID, 3. 1974. Drosophila mauritiana n. sp. du groupe melanogaster de I'Ile Maurice. Bull. Soc. ent. de France, 79, 42-46.

WOODRUFF, R. C., SLATKO, B. E. AND THOMPSON, J. N. JR. 1983. Factors affecting mutation rates in natural populations. In Ashburner, M., Carson, H. L. and Thompson, J. N. Jr. (eds) The Genetics and Biology of Drosophila, vol. 3c. Academic Press, London, pp. 37-124. 